\title{
Prevalência simultânea de hipertensão e diabetes em idosos brasileiros: desigualdades individuais e contextuais
}

\author{
Prevalence of concomitant hypertension and diabetes in Brazilian \\ older adults: individual and contextual inequalities
}

Priscila Maria Stolses Bergamo Francisco ${ }^{1}$

Neuber José Segri ${ }^{2}$

Flávia Silva Arbex Borim ${ }^{1}$

Deborah Carvalho Malta ${ }^{3}$

${ }^{1}$ Departamento de Saúde Coletiva, Faculdade de Ciências Médicas, Universidade Estadual de Campinas. R. Tessália Vieira de Camargo 126, Cidade Universitária. 13083-887 Campinas SP Brasil. primaria@fcm.unicamp.br ${ }^{2}$ Instituto de Ciências Exatas e da Terra,

Departamento de Estatística Universidade Federal de Mato Grosso. Cuiabá MT Brasil.

${ }^{3}$ Coordenação de Doenças e Agravos Não Transmissíveis, Secretaria de Vigilância em Saúde, Ministério da Saúde. Brasília DF Brasil.
Abstract The simultaneous occurrence of specific diseases in older adults is explored little in the literature. The aim of this study was to estimate the prevalence of concomitant hypertension and diabetes in Brazilian older adults and determine associated contextual and individual factors. A population-based cross-sectional study was conducted with 10,991 older adults ( $\geq 60$ years of age) interviewed during the 2012 Vigitel Survey. Statistical analysis involved multi-level Poisson regression. Mean age was 69.4. The prevalence of the concomitant occurrence of hypertension and diabetes was $16.2 \%$, with differences found among the capital cities. Higher prevalence rates were found in cities located in the southern, southeastern and central-western regions of the country as well as among black and brown older adults, those with up to eight years of schooling, non-smokers, ex-smokers and those with excess weight. The effect of geographic region was demonstrated by the $23.5 \%$ increase in the prevalence of the concomitant diseases after adjusting for individual variables. The present findings enabled gauging the effect of context (region of residence) on the prevalence of the main diseases related to an increased mortality rate in the older population.

Key words Epidemiological surveys, Arterial hypertension, Diabetes mellitus, Elder health
Resumo A prevalência da simultaneidade de doenças específicas nos idosos ainda é pouco descrita na literatura. O objetivo do estudo foi estimar a prevalência simultânea de hipertensão arterial e diabetes mellitus em idosos brasileiros, e os fatores contextuais e individuais a ela associados. Estudo transversal de base populacional com idosos ( $\geq 60$ anos) entrevistados pelo Vigitel em $2012(n=10.991)$. As análises foram realizadas por meio de regressão de Poisson multinivel no Stata 12. A média de idade dos idosos foi de 69,4 anos e a prevalência simultânea das doenças foi de $16,2 \%$ com variação nas capitais brasileiras. Em São Paulo e Curitiba as prevalências foram mais elevadas do que em Boa Vista e Manaus. Maiores prevalências foram observadas nas capitais das regiões Sul/Sudeste/Centro-Oeste, nos idosos de cor preta e parda, naqueles com escolaridade $\leq 8$ anos de estudo, nos não fumantes e ex-fumantes, e com excesso de peso. Verificou-se o efeito da região geográfica na prevalência simultânea pela elevação de $23,5 \%$ na magnitude da razão de prevalencia, após ajuste para todas as variáveis individuais. Por meio do estudo, foi possivel dimensionar o efeito do contexto onde estão inseridos os idosos (região de residência) sobre a prevalência das principais doenças que acometem e relacionam-se à mortalidade na população idosa na atualidade. Palavras-chave Inquéritos epidemiológicos, $\mathrm{Hi}$ pertensão arterial, Diabetes mellitus, Saúde do idoso 


\section{Introdução}

Dentre os cinco principais riscos globais para a mortalidade no mundo, se encontram a hipertensão arterial sistêmica e o diabetes mellitus ${ }^{1}$, reconhecidos fatores de risco para as doenças cardiovasculares e causas de óbito na população idosa $^{2,3}$. Com elevadas prevalências, destacam-se entre os principais problemas de saúde pública na atualidade ${ }^{1}$, por sua relação com a morbimortalidade e, principalmente, com as grandes síndromes geriátricas ${ }^{2,3}$.

A hipertensão arterial sistêmica é a mais frequente das doenças cardiovasculares levando à diminuição na qualidade e expectativa de vida da população. Considerada uma condição clínica multifatorial, caracterizada por níveis elevados e sustentados de pressão arterial (PA), frequentemente associa-se a alterações funcionais e/ou estruturais dos órgãos-alvo (coração, encéfalo, rins e vasos sanguíneos) e a alterações metabólicas $^{2}$. Existe uma relação direta da PA com a idade. No Brasil, observam-se prevalências em torno de $50 \%$ para a população idosa ${ }^{4-9}$.

O diabetes mellitus (DM) é um grupo heterogêneo de distúrbios metabólicos que apresenta em comum a hiperglicemia, decorrente de defeitos na ação da insulina, na secreção de insulina ou em ambas. Essa desordem crônica no metabolismo de glicose, com aumento persistente da glicemia, pode desencadear complicações agudas ou crônicas no sistema cardiovascular, renal e neurológico ${ }^{3,10}$. O diabetes mellitus tipo 2 é responsável por 90 a $95 \%$ dos casos desta doença ${ }^{3}$. Estimativas apontam que entre 2010 e 2030 haverá um aumento de $69 \%$ no número de adultos com diabetes nos países em desenvolvimento e de $20 \%$ nos países desenvolvidos ${ }^{11}$. No Brasil, dados da Pesquisa Nacional de Saúde (PNS) estimam em cerca de 9,2 milhões de brasileiros com o diagnóstico de diabetes mellitus, ou 6,2\% da população acima de 18 anos $^{12}$, sendo crescente a prevalência conforme aumenta a idade ${ }^{12,13}$. Na população idosa brasileira, dados da PNAD apontavam prevalência de diabetes acima de $15 \%$, em 2008. De acordo com a PNS, em 2013, a prevalência era de 19,9\% e 19,6\% entre idosos de 65 a 74 anos e acima de 75 anos, respectivamente $^{12}$. Para a população com idade $\geq 60$ anos, estudos em localidades específicas têm revelado prevalências superiores a $13 \% \%^{4,7,14-16}$.

O aumento da importância dessas condições de saúde de um modo geral, e entre os idosos, se reflete fortemente no aumento da procura por serviços, no elevado uso de medicamentos nesta faixa etária ${ }^{4,7}$ e na restrição de atividades pelos acometidos por tais doenças com impacto social importante ${ }^{17,18}$, sendo apontado grau intenso e muito intenso de limitações de atividades diárias em 7,0\% dos diabeticos, e relato de internação em 13,4\% nos últimos 12 meses $^{18}$. Esse aumento da procura por serviços de saúde, tanto na atenção primária, quanto nos serviços de maior complexidade, representa elevado ônus ao Sistema Único de Saúde (SUS), impondo-lhe a necessidade de (re)organização, qualificação e ampliação do atendimento ${ }^{19}$, a partir do conhecimento da demanda.

Principalmente quando associadas, a hipertensão arterial e o diabetes mellitus são relevantes causas de morbidade e mortalidade, com maior risco de doença renal, doença cardíaca coronariana, acidente vascular encefálico e insuficiência cardíaca. Também se associam com comorbidades como a dislipidemia, estado pró-trombótico e disfunção autonômica cardíaca ${ }^{20}$. Estudos mostram que a prevalência de hipertensão é aproximadamente o dobro entre os diabéticos em comparação com os não diabéticos, e o risco de doença cardiovascular é cerca de quatro vezes maior em pacientes com ambas as doenças ${ }^{21,22}$.

O aumento da proporção de idosos na população, observado tanto nos países desenvolvidos quanto naqueles em desenvolvimento, tem sido desigual entre as regiões brasileiras ${ }^{23}$. A ocorrência de doenças e sua distribuição na sociedade decorre de fortes processos de determinação social, econômica, cultural, ambiental, política, entre outros, além de fatores individuais, tais como as características sociodemográficas e fatores comportamentais ${ }^{24}$.

O Brasil é um país de dimensões continentais com situações sociodemográficas e epidemiológicas contrastantes nas diferentes regiõos s, $, 8,12,23^{2}$ e, considerando-se que o risco cardiovascular aumenta progressivamente com o número de fatores de risco presentes, a distribuição da hipertensão e diabetes simultaneamente na população estudada, caracteriza situação de maior risco para as doencas cardiovasculares ${ }^{21}$. O objetivo do presente estudo foi estimar a prevalência simultânea de hipertensão arterial e diabetes mellitus em idosos brasileiros, e os fatores contextuais e individuais associados, para identificar desigualdades na distribuição desses fatores.

\section{Método}

Neste estudo foram avaliados dados provenientes do Vigitel relativos ao ano de 2012. Este inquérito 
telefônico vem sendo realizado anualmente pelo Ministério da Saúde desde 2006, nas capitais brasileiras e no Distrito Federal. O sistema seleciona uma amostra da população adulta ( $\geq 18$ anos) residente em domicílios servidos por pelo menos uma linha de telefone fixo.

Trata-se de um estudo transversal de base populacional com amostra probabilística em três estágios: a primeira etapa da amostragem consistiu no sorteio sistemático de 5 mil linhas telefônicas divididas em réplicas ou subamostras de 200 linhas cada, reproduzindo a mesma proporção de linhas por região da cidade ou prefixo telefônico. O segundo estágio, realizado concomitantemente às entrevistas, incluiu a identificação de linhas efetivamente residenciais e ativas, as quais foram consideradas elegíveis para o sistema. Nessa etapa excluíram-se as linhas empresariais, fora de serviço ou inexistentes, assim como os números que não responderam a seis ligações realizadas em horários/dias diferentes. Identificadas as linhas elegíveis, no terceiro estágio sortearam-se os indivíduos para entrevista, dentre todos os adultos ( $\geq 18$ anos) residentes no domicílio ${ }^{25}$.

Também no ano de 2012, uma amostra mínima de 2000 entrevistas por cidade foi considerada, para que se pudesse estimar com nível de 95\% de confiança e erro máximo de cerca de 2 pontos percentuais, a frequência de qualquer fator de risco para doenças crônicas na população adulta e idosa. Para compensar o viés da não cobertura universal de telefonia fixa, utilizaram-se fatores de ponderação. As estimativas produzidas foram ajustadas à população adulta brasileira, a partir do uso de um peso pós-estratificação, calculado com base em 36 categorias de análise por sexo (feminino e masculino), faixa etária (18-24, 25-34, 35-44, 45-54, 55-64 e $\geq 65$ anos) e nível de instrução (sem instrução ou fundamental incompleto, fundamental completo ou médio incompleto, médio completo ou superior incompleto e superior completo). O peso pós-estratificação de cada indivíduo da amostra foi calculado pelo método ' $r a k e^{\text {'25. }}$.

O instrumento de coleta de dados do Vigitel aborda características demográficas e socioeconômicas dos indivíduos, informações sobre hábito alimentar, atividade física, peso e altura recordados, frequência de consumo de cigarros e de bebidas alcoólicas, autoavaliação de saúde, uso de medicamentos entre outras questões, como o diagnóstico médico anterior de algumas doenças. Neste estudo a presença simultânea das doenças foi verificada por meio das seguintes perguntas: Algum médico já lhe disse que o(a) Sr(a) tem pres- são alta?. E em relação ao diabetes: Algum médico já the disse que o(a) Sr(a) tem diabetes? A partir das respostas obtidas para os idosos (sim ou não) uma variável dicotômica foi criada, considerando-se presença simultânea a referência positiva do entrevistado em relação a ambas as questões.

Neste estudo foram utilizadas como variáveis independentes a macrorregião geográfica do país (Norte/Nordeste e Sul/Sudeste/Centro-Oeste), sexo (masculino e feminino), faixa etária (60 a 64, 65 a 69, 70 a 74, 75 a 79 e $\geq 80$ anos), situação conjugal (solteiro, casado/unido, viúvo e separado/divorciado), cor da pele/raça (branca, parda, preta ou amarela/indígena), escolaridade ( 0 a 4 , 5 a 8 e 9 ou mais anos de estudo) e posse de plano de saúde ( $\operatorname{sim}$ ou não). As variáveis relacionadas ao comportamento foram tabagismo (não fumante, ex-fumante e fumante atual), índice de massa corporal $\left(<22 \mathrm{Kg} / \mathrm{m}^{2}, 22\right.$ a $27 \mathrm{Kg} / \mathrm{m}^{2}$ e $>$ $\left.27 \mathrm{Kg} / \mathrm{m}^{2}\right)^{26}$ e prática de atividade física ( $\operatorname{sim}$ ou não). O recorte para a análise geográfica, ou seja, entre as regiões Norte/Nordeste e Sul/Sudeste/ Centro-Oeste considerou o Índice de Desenvolvimento Humano (IDH) das capitais segundo o Censo de $2010^{27}$.

A caracterização da população estudada foi realizada por meio das frequências relativas. As associações entre as variáveis e a presença das doenças foram verificadas pelo teste Qui-quadrado de Pearson com correção de segunda ordem (Rao \& Scott), considerando-se um nível de significância de $5 \%$. As razões de prevalência e os respectivos intervalos de confiança de $95 \%$ foram estimados pela regressão de Poisson com variância robusta. Em todas as análises considerou-se o efeito do desenho amostral para análise de inquéritos baseados em delineamentos complexos do programa Stata 12.0.

A análise dos fatores associados à prevalência simultânea de hipertensão e diabetes foi realizada por meio de regressão de Poisson multinível, usando o modelo de efeitos fixos e intercepto randômico que possibilita examinar grupos e indivíduos inseridos (dentro) nesses grupos, simultaneamente, considerando a variável resposta medida no nível individual e as variáveis explicativas medidas tanto no nível dos indivíduos, quanto nos agregados aos quais pertencem ${ }^{28}$. No presente estudo foram considerados dois níveis de organização dos dados, quais sejam: macrorregião do país e os idosos entrevistados.

No modelo teórico para investigação dos fatores contextuais e individuais associados à prevalência simultânea de hipertensão arterial e diabetes mellitus em idosos, considerou-se a 
macrorregião do país como variável contextual. Sexo, idade, situação conjugal e cor da pele/ raça representaram as variáveis demográficas. A condição socioeconômica foi avaliada pela escolaridade e posse de plano de saúde, consideradas proxy dessa condição e, os comportamentos relacionados à saúde, foram mensurados por meio das variáveis tabagismo, índice de massa corporal e prática de atividade física. A análise de regressão multinível levou em conta o modelo teórico de determinação do desfecho apresentado na Figura 1.

Inicialmente, foi realizada análise de regressão de Poisson múltipla hierarquizada. Na primeira etapa foram incluídas as variáveis relativas às características demográficas (sexo, faixa etária, estado civil e cor da pele/raça), ajustadas entre si e pela variável do nível 1 (região de residência). Em seguida, foram inseridas as variáveis socio-

Nível 1

$$
\text { Região Geográfica }
$$

Sexo

Faixas etárias

Situação Conjugal

Cor da pele/raça

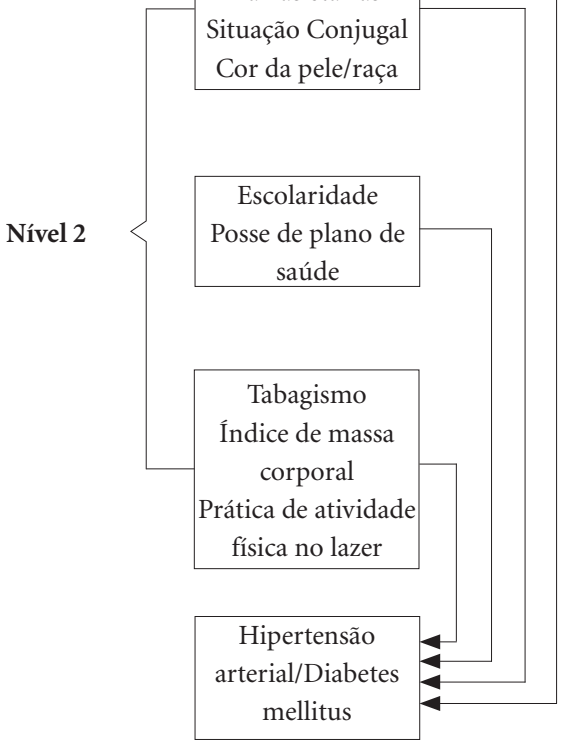

Figura 1. Modelo teórico para investigação dos fatores contextuais e individuais associados à prevalência simultânea de hipertensão arterial e diabetes mellitus em idosos, estruturado em níveis hierárquicos. econômicas (escolaridade e posse de plano de saúde) e mantidas aquelas que apresentaram um nível descritivo inferior a 0,25 quando ajustadas pelas variáveis demográficas, do mesmo bloco hierárquico e pela variável de contexto. Finalmente, as variáveis relacionadas aos comportamentos de saúde (tabagismo, IMC e prática de atividade física) foram inseridas no modelo e permaneceram aquelas ajustadas por todas as do mesmo nível (individual) e pela macrorregião de residência, que apresentaram significância estatística $(\mathrm{p}<0,05)$.

O sexo e a idade permaneceram como variáveis de ajuste em todas as etapas, considerandose que as regiões brasileiras apresentam diferenças em relação à distribuição da população idosa por sexo e faixas etárias. Ainda, verificam-se diferenciais entre os homens e mulheres no risco de adoecer, na percepção de sinais e sintomas, procura por serviços de saúde e no modo de cuidado $^{8,29}$. No que se refere à prevalência de doenças crônicas, deve-se considerar que o padrão de distribuição por idade diferencia-se conforme o tipo de problema de saúde ${ }^{8}$.

Desse modo, a cada etapa, obteve-se um efeito composicional, ou seja, de fatores individuais sobre o desfecho individual (presença simultânea de hipertensão e diabetes), considerando-se o efeito da macrorregião de residência a que os idosos pertenciam. Portanto, as razões de prevalência foram ajustadas pelas variáveis situadas no mesmo nível de determinação (individual) e pela variável situada no nível mais distal (contexto). Por fim, ajustou-se a variável contextual - macrorregião - pelas variáveis que representavam as características individuais estatisticamente associadas ao desfecho.

Os indivíduos foram informados sobre os objetivos da pesquisa no momento do contato telefônico e o consentimento livre e esclarecido foi substituído pelo consentimento verbal. O estudo foi aprovado pela Comissão Nacional de Ética em Pesquisa em Seres Humanos, do Ministério da Saúde.

\section{Resultados}

No ano de 2012, para o conjunto das 27 cidades, o Vigitel realizou 45.448 entrevistas com adultos ( $\geq 18$ anos). A taxa de participação foi de $64,9 \%$ (variando entre 60,8\%, em Belém, e 73,1\%, em Palmas). As recusas em participar do sistema de monitoramento ocorreram no contato inicial com o domicílio, ou após o sorteio do indivíduo a 
ser entrevistado, e corresponderam a 5,9\% das linhas elegíveis. No referido ano, o tempo médio de duração das entrevistas foi de aproximadamente 11 minutos, variando entre 5 e 58 minutos.

Entre os adultos, 10.991 dos entrevistados pelo inquérito telefônico eram idosos. A prevalência simultânea de hipertensão arterial e diabetes mellitus foi de 16,2 ( IC $_{95 \%}: 14,8$ - 17,7) e apresentou variação nas capitais brasileiras. Observaramse maiores prevalências em São Paulo e Curitiba, em relação à Boa Vista e Manaus (Figura 2).

A Tabela 1 apresenta a distribuição da população idosa e a prevalência das doenças, segundo as macrorregiões do país e características indivi- duais consideradas. Maiores prevalências foram observadas nas capitais das regiões Sul, Sudeste e Centro-Oeste, nos idosos de cor da pele/raça preta e amarela/indígena, naqueles com menor escolaridade ( $\leq 8$ anos de estudo), nos não fumantes e ex-fumantes, e nos que estavam com excesso de peso à época da pesquisa. Não houve associação entre a inatividade física e a presença de ambas as doenças ( $\left.\mathrm{RP}=1,20 ; \mathrm{IC}_{95 \%}: 1,00-1,44\right)$.

Entre os idosos residentes nas regiões Sul, Sudeste e Centro-Oeste, a prevalência simultânea de hipertensão arterial e diabetes mellitus foi $17,0 \%$ maior do que naqueles residentes nas regiões Norte e Nordeste. As análises ajustadas

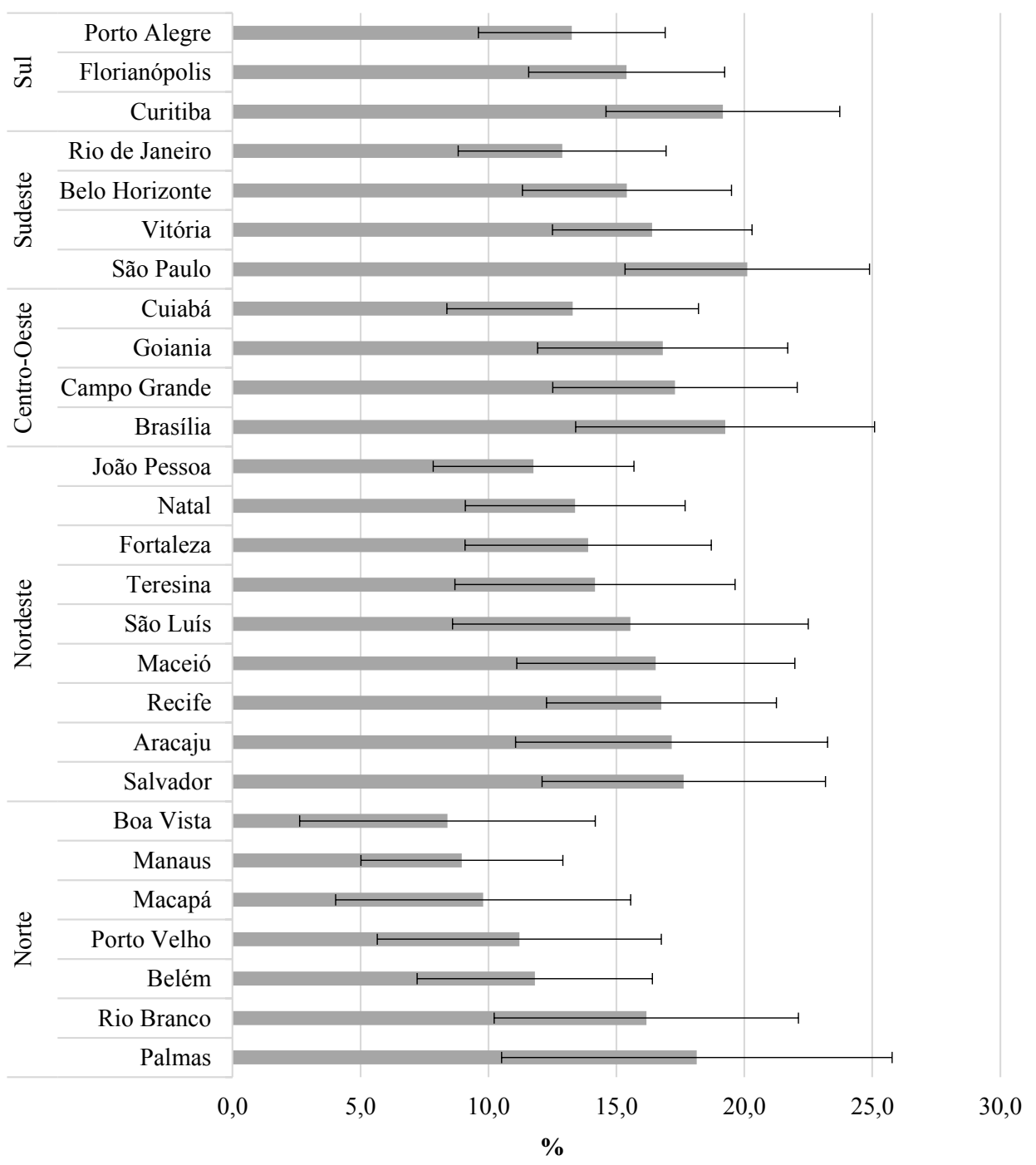

Figura 2. Prevalência simultânea de hipertensão arterial e diabetes mellitus em idosos nas capitais brasileiras. Vigitel, Brasil, 2012. 
Tabela 1. Prevalência simultânea de hipertensão arterial e diabetes mellitus em idosos segundo região geográfica, características demográficas, socioeconômicas e comportamentais. Vigitel, Brasil, 2012.

\begin{tabular}{|c|c|c|c|}
\hline Variáveis/categorias & Frequência (\%) & Prevalência (\%) & $\mathbf{p}^{*}$ \\
\hline \multicolumn{4}{|l|}{$1^{\circ}$ nível: contexto } \\
\hline \multicolumn{4}{|l|}{ Região geográfica } \\
\hline Macrorregião & & & 0,041 \\
\hline Norte e Nordeste & 29,4 & 14,5 & \\
\hline Sul, Sudeste e Centro-Oeste & 70,6 & 17,0 & \\
\hline \multicolumn{4}{|l|}{$2^{\circ}$ nível: indivíduos } \\
\hline \multicolumn{4}{|l|}{ Características demográficas } \\
\hline Sexo & & & 0,175 \\
\hline Masculino & 39,4 & 15,0 & \\
\hline Feminino & 60,6 & 17,1 & \\
\hline Faixa etária (anos) & & & 0,444 \\
\hline $60-64$ & 33,5 & 14,7 & \\
\hline $65-69$ & 22,7 & 17,9 & \\
\hline $70-74$ & 18,0 & 17,2 & \\
\hline $75-79$ & 14,1 & 17,5 & \\
\hline 80 e mais & 11,7 & 15,0 & \\
\hline Estado civil & & & 0,301 \\
\hline Solteiro & 10,6 & 13,4 & \\
\hline Casado/unido & 57,6 & 16,1 & \\
\hline Viúvo & 23,8 & 18,2 & \\
\hline Separado/divorciado & 8,0 & 16,9 & \\
\hline Cor da pele/Raça & & & 0,035 \\
\hline Branca & 56,3 & 15,2 & \\
\hline Parda & 28,5 & 15,4 & \\
\hline Preta & 9,8 & 20,8 & \\
\hline Amarela/indígena & 5,4 & 23,6 & \\
\hline \multicolumn{4}{|l|}{ Socioeconômicas } \\
\hline Escolaridade (anos de estudo) & & & 0,016 \\
\hline$\leq 4$ & 43,7 & 17,8 & \\
\hline $5-8$ & 24,1 & 17,5 & \\
\hline$\geq 9$ & 32,2 & 13,3 & \\
\hline Plano privado de saúde & & & 0,133 \\
\hline Não possui & 49,6 & 17,5 & \\
\hline Possui & 50,4 & 15,2 & \\
\hline \multicolumn{4}{|l|}{ Comportamento } \\
\hline Tabagismo & & & $<0,001$ \\
\hline Não fumante & 55,2 & 17,1 & \\
\hline Ex-fumante & 35,6 & 17,4 & \\
\hline Fumante & 9,2 & 7,6 & \\
\hline Índice de massa corporal & & & $<0,001$ \\
\hline$<22 \mathrm{Kg} / \mathrm{m}^{2}$ & 12,1 & 8,8 & \\
\hline 22 a $27 \mathrm{Kg} / \mathrm{m}^{2}$ & 39,6 & 12,9 & \\
\hline$>27 \mathrm{Kg} / \mathrm{m}^{2}$ & 48,3 & 21,0 & \\
\hline Inatividade física & & & 0,051 \\
\hline Não & 69,2 & 15,4 & \\
\hline Sim & 30,8 & 18,4 & \\
\hline
\end{tabular}

Valor de p do teste Qui-quadrado com correção de Rao-Scott.

evidenciaram desigualdades individuais: a prevalência foi $48,0 \%$ e 55,0\% maior nos de cor da pele parda e preta, respectivamente. Observaram-se também maiores prevalências naqueles com es- colaridade $\leq 8$ anos de estudo e particularmente elevada entre os idosos com excesso de peso (RP $=2,63$; IC $85 \%: 1,82-3,80)$. Entre os fumantes, a prevalência foi significativamente menor $(\mathrm{RP}=$ 
0,45; IC95\%: 0,27 - 0,74), ou seja, entre os idosos tabagistas, observou-se, em 2012, uma menor proporção de indivíduos acometidos por essas condições (Tabela 2).
As razões de prevalência da variável de contexto - região geográfica - antes e após o ajuste pelas variáveis individuais são apresentadas na Tabela 3. Observa-se que houve uma elevação

Tabela 2. Análise multinível de fatores associados à prevalência simultânea de hipertensão arterial e diabetes mellitus em idosos. Vigitel, Brasil, 2012.

\begin{tabular}{|c|c|c|c|c|}
\hline Variáveis/categorias & $\mathbf{R P}_{\text {bruta }}\left(\mathbf{I C}_{95 \%}\right)$ & $\mathbf{p}$ & $\mathbf{R P}$ ajustada $\left(\right.$ IC $\left._{95 \%}\right)$ & p \\
\hline $1^{\circ}$ nível: contexto & & & & \\
\hline $\begin{array}{l}\text { Região geográfica } \\
\text { Norte e Nordeste } \\
\text { Sul, Sudeste e Centro-Oeste }\end{array}$ & $\begin{array}{c}1 \\
1,17(1,01-1,36)\end{array}$ & 0,040 & & \\
\hline $2^{\circ}$ nível: indivíduos & & & & \\
\hline $\begin{array}{l}\text { Gênero } \\
\text { Masculino } \\
\text { Feminino }\end{array}$ & $\begin{array}{c}1 \\
1,14(0,94-1,39)\end{array}$ & 0,177 & $\begin{array}{c}1 \\
1,11(0,87-1,41)\end{array}$ & 0,404 \\
\hline $\begin{array}{l}\text { Faixa etária (anos) } \\
\quad 60-64 \\
65-69 \\
70-74 \\
75-79 \\
80 \text { e mais }\end{array}$ & $\begin{array}{c}1 \\
1,22(0,95-1,57) \\
1,17(0,91-1,51) \\
1,19(0,89-1,60) \\
1,02(0,76-1,39)\end{array}$ & $\begin{array}{l}0,126 \\
0,219 \\
0,233 \\
0,871\end{array}$ & $\begin{array}{c}1 \\
1,27(0,97-1,67) \\
1,21(0,91-1,60) \\
1,26(0,90-1,76) \\
0,98(0,66-1,45)\end{array}$ & $\begin{array}{l}0,087 \\
0,187 \\
0,176 \\
0,915\end{array}$ \\
\hline $\begin{array}{l}\text { Estado civil } \\
\text { Solteiro } \\
\text { Casado/unido } \\
\text { Viúvo } \\
\text { Separado/divorciado }\end{array}$ & $\begin{array}{c}1 \\
1,20(0,87-1,64) \\
1,35(0,97-1,88) \\
1,26(0,84-1,87)\end{array}$ & $\begin{array}{l}0,269 \\
0,072 \\
0,257\end{array}$ & $\begin{array}{c}1 \\
1,46(1,03-2,08) \\
1,50(1,02-2,20) \\
1,41(0,92-2,17)\end{array}$ & $\begin{array}{l}\mathbf{0 , 0 3 5} \\
\mathbf{0 , 0 3 9} \\
0,112\end{array}$ \\
\hline $\begin{array}{l}\text { Cor da pele/Raça } \\
\text { Branca } \\
\text { Parda } \\
\text { Preta } \\
\text { Amarela/indígena }\end{array}$ & $\begin{array}{c}1 \\
1,01(0,82-1,25) \\
1,36(0,99-1,88) \\
1,55(1,07-2,23)\end{array}$ & $\begin{array}{l}0,912 \\
0,057 \\
0,020\end{array}$ & $\begin{array}{c}1 \\
1,48(1,07-2,04) \\
1,55(1,08-2,23) \\
1,07(0,86-1,34)\end{array}$ & $\begin{array}{l}\mathbf{0 , 0 1 8} \\
\mathbf{0 , 0 1 8} \\
0,511\end{array}$ \\
\hline $\begin{array}{l}\text { Socioeconômica }^{* *} \\
\text { Escolaridade (anos de estudo) }\end{array}$ & & & & \\
\hline $\begin{array}{l}\geq 9 \\
5-8 \\
\leq 4\end{array}$ & $\begin{array}{c}1 \\
1,31(1,04-1,67) \\
1,34(1,10-1,65)\end{array}$ & $\begin{array}{l}0,021 \\
0,004\end{array}$ & $\begin{array}{c}1 \\
1,31(1,00-1,71) \\
1,40(1,09-1,81)\end{array}$ & $\begin{array}{l}0,047 \\
0,009\end{array}$ \\
\hline $\begin{array}{l}\text { Plano privado de saúde } \\
\text { Não possui } \\
\text { Possui }\end{array}$ & $\begin{array}{c}1 \\
1,07(0,98-1,17)\end{array}$ & 0,133 & $\begin{array}{c}1 \\
1,01(0,91-1,13)\end{array}$ & 0,840 \\
\hline $\begin{array}{l}\text { Comportamento }^{* * *} \\
\text { Tabagismo }\end{array}$ & & & & \\
\hline $\begin{array}{l}\text { Não fumante } \\
\text { Ex-fumante } \\
\text { Fumante }\end{array}$ & $\begin{array}{c}1 \\
1,02(0,85-1,23) \\
0,45(0,29-0,68)\end{array}$ & $\begin{array}{c}0,846 \\
<0,001\end{array}$ & $\begin{array}{c}1 \\
1,06(0,87-1,30) \\
0,45(0,27-0,74)\end{array}$ & $\begin{array}{l}0,539 \\
\mathbf{0 , 0 0 2}\end{array}$ \\
\hline $\begin{array}{l}\text { Índice de massa corporal } \\
<22 \mathrm{Kg} / \mathrm{m}^{2}\end{array}$ & 1 & & 1 & \\
\hline $\begin{array}{l}22 \text { a } 27 \mathrm{Kg} / \mathrm{m}^{2} \\
>27 \mathrm{Kg} / \mathrm{m}^{2}\end{array}$ & $\begin{array}{l}1,47(1,02-2,12) \\
2,40(1,69-3,40)\end{array}$ & $\begin{array}{c}0,040 \\
<0,001\end{array}$ & $\begin{array}{l}1,66(1,12-2,44) \\
2,63(1,82-3,80)\end{array}$ & $\begin{array}{c}0,011 \\
<0,001\end{array}$ \\
\hline $\begin{array}{l}\text { Inatividade física } \\
\text { Não } \\
\text { Sim }\end{array}$ & $\begin{array}{c}1 \\
1,20(1,00-1,44)\end{array}$ & 0,050 & $\begin{array}{c}1 \\
1,18(0,90-1,55)\end{array}$ & 0,231 \\
\hline
\end{tabular}


Tabela 3. Elevação percentual do efeito da região geográfica na prevalência simultânea de hipertensão arterial e diabetes mellitus em idosos, após ajuste pelos fatores demográficos, socioeconômicos e comportamentais. Vigitel, Brasil, 2012.

\begin{tabular}{|c|c|c|c|c|}
\hline Variável contextual & $\begin{array}{l}\mathrm{RP}_{\text {bruta }} \\
\left(\mathrm{IC}_{95 \%}\right)\end{array}$ & $\begin{array}{c}\mathbf{R P}_{\text {ajustada }}{ }^{*} \\
\left(\mathrm{IC}_{95 \%}\right)\end{array}$ & Elevação $^{* *}(\%)$ & $\operatorname{Deff}^{* *}$ \\
\hline \multicolumn{5}{|l|}{ Região geográfica } \\
\hline Norte e Nordeste & 1 & 1 & & \\
\hline Sul, Sudeste e Centro-Oeste & $1,17(1,01-1,36)$ & $1,21(1,03-1,44)$ & 23,5 & 2,25 \\
\hline
\end{tabular}

de $23,5 \%$ na magnitude da razão de prevalência bruta que passou de 1,17 para 1,21 após ajuste pelas variáveis individuais, indicando que esta variável permaneceu associada à presença simultânea de hipertensão arterial e diabetes mellitus independentemente das variáveis individuais.

\section{Discussão}

A prevalência simultânea de hipertensão arterial e diabetes mellitus nos idosos foi de 16,2\% e apresentou variação nas capitais brasileiras. Sabe-se que a prevalência de doenças crônicas entre os idosos é elevada ${ }^{8,30,31}$. No Estudo Bambuí, 69\% dos idosos relataram ter pelo menos uma doença crônica ${ }^{30}$ e no Cardiovascular Health Study (CHS) apenas 18,5\% dos idosos não referiram essa condição ${ }^{31}$. Em estudo que avaliou as fontes de obtenção de medicamentos para hipertensão e diabetes no Brasil com dados do Vigitel de 2011, a prevalência de hipertensão arterial variou de $57,1 \%$ a $60,3 \%$ nas faixas etárias de 60 a 69 e $\geq$ 70 anos, respectivamente. Em relação ao diabetes mellitus, os valores observados para esses subgrupos foram de $18,8 \%$ e $22,0 \%{ }^{4}$.

A prevalência da simultaneidade de doenças específicas entre os idosos ainda é pouco descrita na literatura nacional. Freitas e Garcia ${ }^{32}$, com dados da Pesquisa Nacional por Amostra de Domicílios (PNAD) de 1998, 2003 e 2008 revelaram a rápida elevação das prevalências simultâneas no período: a partir dos 50 anos de idade, observaram-se elevações significativas nas regiões brasileiras e, entre idosos brasileiros com idade $\geq 65$ anos, as prevalências encontraram-se acima de 15\%. Estudo com 297 usuários cadastrados no Plano de Reorganização da Atenção à Hipertensão Arterial e ao Diabetes Mellitus (HIPERDIA/ MS) de um Centro de Saúde da Família da Secretaria Executiva Regional VI (SER VI) de Fortaleza-Ceará em 2009, verificou proporção de
19,5\% de ambas as doenças ${ }^{33}$. Entre os idosos integrantes da linha-base do Projeto Bambuí (n $=1.606$ ), 942 eram hipertensos e/ou diabéticos. Destes 77,5\% eram apenas hipertensos, 6,6\% eram somente diabéticos e $15,9 \%$ eram hipertensos e diabéticos ${ }^{34}$. Diferentemente do presente estudo, os citados não apresentaram intervalos de confiança associados às estimativas pontuais da prevalência simultânea das doenças, impossibilitando comparações.

Neste estudo, os fatores individuais associados à prevalência simultânea das doenças consideradas corroboram com aqueles verificados em vários outros estudos relacionados à hipertensão arterial e ao diabetes mellitus s,9,14-16. $^{6}$.

A escolaridade e a raça são determinantes sociais das condições de saúde e doença dos ido$\operatorname{sos}^{35}$. Dados nacionais e internacionais revelam desigualdades de escolaridade e raça em relação à presença de doenças crônicas ${ }^{8,36,37}$, o que pode decorrer da associação com a falta de acesso a oportunidades sociais ao longo da vida, aumentando a vulnerabilidade desse subgrupo a desfechos desfavoráveis na velhice. Particularmente, a cor da pele/raça se mostra como importante variável na investigação da desigualdade social e aspectos de saúde ${ }^{2,5,38,39}$. Estratégias direcionadas à diminuição dessas desigualdades podem ter impacto na redução da carga das doenças crônicas nos segmentos populacionais mais vulneráveis.

O tabagismo apresentou associação inversa com a prevalência simultânea das doenças. Associações significativas e inversas de tabagismo e hipertensão arterial foram verificadas entre idosos do Rio Grande do Sul ${ }^{40}$, em Campinas ${ }^{41}$, e em estudo entre idosos utilizando subamostra do Vigitel ${ }^{42}$. O tabagismo é o principal fator de risco para mortalidade por doenças crônicas não transmissíveis $(\mathrm{DCNT})^{43}$ e a recomendação para sua cessação entre pessoas acometidas por tais doenças deve ser uma medida imediata. Embora a cessação do tabagismo não reduza a pressão 
arterial, o abandono do tabaco é, provavelmente, a medida isolada mais efetiva para a redução do risco de doenças cardiovasculares ${ }^{42}$.

De acordo com as diretrizes para a cessação de tabagismo ${ }^{44}$, os danos causados pelo fumo podem ser reduzidos mesmo nos que fumaram três décadas ou mais, permitindo melhor controle da evolução de doenças pré-existentes e elevação da expectativa de vida. A menor prevalência de tabagistas hipertensos e diabéticos, verificada neste estudo pode ser parcialmente explicada pelo fato de que para o acompanhamento dos níveis pressóricos e glicêmicos, o idoso teria maior contato com profissionais de saúde para recomendações e intervenções educativas. Estes resultados corroboram com a hipótese de que o diagnóstico da hipertensão tenha levado à cessação do tabagismo ${ }^{42}$.

No que se refere à associação entre o IMC $\left(\geq 22 \mathrm{Kg} / \mathrm{m}^{2}\right)$ e as doenças, ressalta-se que medidas não farmacológicas são fundamentais para a prevenção e controle da pressão arterial e do diabetes mellitus. Estudos apontam que associada à dieta alimentar, a atividade física pode ser benéfica para auxiliar no controle pressórico e glicêmico, na redução ponderal, no aumento da massa muscular e na redução de eventos cardiovasculares mais graves ${ }^{2,3}$.

As desigualdades regionais observadas em vários indicadores sociodemográficos e de saú$\mathrm{de}^{5,8,23}$ também foram verificadas em relação à prevalência das doenças mais frequentes na população idosa. Nas regiões geográficas com maior IDH (Sul, Sudeste e Centro-Oeste) observou-se maior prevalência simultânea das doenças, denotando a influência do contexto no desfecho investigado.

Deve-se destacar que a diversidade de características individuais resulta de forças biológicas e sociais que favorecem ou limitam a ocorrência de muitas doenças ${ }^{45}$. Fatores sociais, econômicos, culturais, raciais e comportamentais são determinantes de vários desfechos em saúde e apresentam-se interrelacionados e sobrepostos de forma hierárquica ${ }^{46,47}$. Segundo Goldstein ${ }^{48}$, o modelo multinível pode ser visto como um sistema hierárquico de equações de regressão, possibilitando a estimação dos efeitos intragrupos (efeitos individuais) e dos efeitos entre grupos (efeitos contextuais).

Ressalta-se ainda que as disparidades regionais observadas para a prevalência simultânea das doenças podem refletir menor acesso ao diagnóstico médico, já que a morbidade referida também depende do acesso e uso dos serviços médicos moldando-se, tanto pela capacidade de procura e obtenção do cuidado, quanto pela organização da atenção que define a oferta, a qualidade e a resolubilidade das ações serviços ${ }^{7,8}$.

Com o deslocamento em bloco da distribuição populacional, e não em função apenas do número de indivíduos na faixa etária de risco, a sobreposição de problemas crônicos de saúde se torna progressivamente mais importante porque demanda atenção qualificada. No âmbito da prevenção primária pode-se, por meio de ações de promoção de saúde, postergar o surgimento dessas doenças mesmo em idades avançadas. Secundariamente, o diagnóstico precoce possibilita, além da oferta e do uso de medicamentos adequados ao tratamento, recomendações e medidas educativas para os idosos hipertensos e diabéticos, com impacto positivo sobre a qualidade e expectativa de vida.

Entre as limitações do presente estudo destaca-se o uso da morbidade autorreferida. A validade da informação referida para hipertensão arterial e diabetes mellitus vem sendo verificada em estudos com a população idosa ${ }^{49}$ ou que consideraram os idosos como subgrupo de análise ${ }^{50,51}$. Particularmente em relação ao diabetes, deve-se considerar que os entrevistados não diferenciaram o tipo de doença, no entanto, cerca de $90 \%$ do total dos casos de diabetes em adultos são do tipo $2^{3}$.

O uso do cadastro de telefones fixos nas capitais para fins de sorteio da amostra também deve ser considerado como uma limitação do estudo. Nas regiões metropolitanas do Sul, Sudeste e Centro-Oeste que apresentam cobertura telefônica acima de 70\%, os vícios devido à exclusão de domicílios sem telefone fixo podem ser considerados desprezíveis ${ }^{52}$. Na comparação de estimativas obtidas de inquérito domiciliar e telefônico para o autorrelato de doenças crônicas, não foram observadas diferenças entre as prevalências para hipertensão e diabetes referidos entre os idosos residentes em Campinas $(\mathrm{SP})^{53}$. Nas regiões Norte e Nordeste que apresentam taxas de cobertura telefônica inferiores a 70\%, para corrigir os vícios potenciais o Vigitel utilizou o método de ponderação por célula segundo sexo, idade e escolaridade, com ajuste da distribuição da amostra às características da população residente em cada capital (de acordo com o Censo do IBGE), para reduzir as diferenças entre a população com e sem telefone $e^{52}$.

Ressalta-se que a promoção da saúde afeta o conjunto da população, no contexto de sua vida diária, e não se concentra nos indivíduos que correm risco de desenvolver doenças específicas ${ }^{45}$. As pre- 
valências observadas são compatíveis com a história natural de ambas as doenças e com o acúmulo de fatores de risco durante o ciclo de vida. Mudança de comportamentos e do estilo de vida da população adulta e idosa é de difícil consolidação. Quanto mais precoces as ações de promoção à saúde, considerando-se as disparidades regionais para a definição de prioridades, maiores as chances de redução do surgimento, também precoce, das principais doenças que acometem os idosos na atualidade.

\section{Conclusões}

A prevalência simultânea de hipertensão arterial e diabetes mellitus nos idosos brasileiros foi superior a 15\%, em 2012, e representa um importante problema de Saúde Pública no Brasil. Além do tratamento farmacológico, a prática de atividade física e a adoção de dietas cardioprotetoras podem diminuir o risco de eventos cardiovascula- res mais graves ou mesmo fatais neste subgrupo populacional.

Mais do que os fatores individuais relacionados ao desfecho avaliado, relações longitudinais entre os determinantes sociais de saúde envolvidos no processo saúde-doença de tais enfermidades podem ser evidenciados ao se analisar a população idosa inserida em contextos diversos. O Brasil possui dimensões continentais e fases distintas de transição epidemiológica, com polarização entre diferentes áreas geográficas e grupos sociais. O monitoramento constante da prevalência simultânea de hipertensão arterial e diabetes mellitus em diferentes localidades é necessário para o planejamento de ações de saúde com ênfase nessas doenças. Por meio da análise realizada, foi possível dimensionar o efeito do contexto onde estão inseridos os idosos (região de residência), sobre a prevalência das principais doenças que acometem e relacionam-se à mortalidade na população idosa.

\section{Colaboradores}

PMSB Francisco elaborou a proposta e redação do artigo, planejou, programou e executou as análises estatísticas e realizou a revisão da literatura. FSA Borim contribuiu na redação do artigo e realizou a revisão da literatura. NJ Segri e DC Malta revisaram as análises estatísticas e contribuiram na revisão crítica do manuscrito. 


\section{Referências}

1. World Health Organization (WHO). Global health risks: mortality and burden of disease attributable to selected major risks. Geneva: WHO; 2009.

2. Sociedade Brasileira de Cardiologia, Sociedade Brasileira de Hipertensão, Sociedade Brasileira de Nefrologia. VI Diretrizes Brasileiras de Hipertensão. Arq Bras Cardiol 2010; 95(1 Supl.1):1-51.

3. Sociedade Brasileira de Diabetes. XII Diretrizes da Sociedade Brasileira de Diabetes 2015-2016. [acessado 2016 Jul 3]. Disponível em: http://www.diabetes. org.br/sbdonline/images/docs/DIRETRIZES-SBD-2015-2016.pdf

4. Costa KS, Francisco PMSB, Malta DC, Barros MBA. Fontes de obtenção de medicamentos para hipertensão e diabetes no Brasil: resultados de inquérito telefônico nas capitais brasileiras e no Distrito Federal, 2011. Cad Saude Publica 2016; 32(2):e00090014.

5. Andrade SSA, Stopa SR, Brito AS, Chueri PS, Szwarcwald CL, Malta DC. Prevalência de hipertensão arterial autorreferida na população brasileira: análise da Pesquisa Nacional de Saúde, 2013. Epidemiologia e Serviços de Saúde 2015; 24(2):297-304.

6. Zattar LC, Boing AF, Giehl MWC, Orsi E. Prevalência e fatores associados à pressão arterial elevada, seu conhecimento e tratamento em idosos no sul do Brasil. Cad Saude Publica 2013; 29(3):507-521.

7. Gontijo MF, Ribeiro AQ, Klein CH, Rozenfeld R, Acurcio FA. Uso de anti hipertensivos e antidiabéticos por idosos: inquérito em Belo Horizonte, Minas Gerais, Brasil. Cad Saude Publica 2012; 28(7):1337-1346.

8. Barros MBA, Francisco PMSB, Lima MG, Cesar CLG. Social inequalities in health among the elderly. Cad Saude Publica 2011; 27(Supl.):S198-S208.

9. Zaitune MPA, Barros MBA, César CLG, Carandina L, Goldbaum M. Hipertensão arterial em idosos: prevalência, fatores associados e práticas de controle no Município de Campinas, São Paulo, Brasil. Cad Saude Publica 2006; 22(2):285-294.

10. American Diabetes Association. Classification and Diagnosis of Diabetes. Diabetes Care 2015; 38(Supl. 1):S8-S16

11. Shaw JE, Sicree RA, Zimmet PZ. Global estimates of the prevalence of diabetes for 2010 and 2030. Diabetes Res Clin Pract 2010; 87(1):4-14.

12. Iser BPM, Stopa SR, Chueiri PS, Szwarcwald CL, Malta DC, Monteiro HOC, Duncan BB, Schmidt MI. Prevalência de diabetes autorreferido no Brasil: resultados da Pesquisa Nacional de Saúde 2013. Epidemiol Serv Saúde 2015; 24(2):305-314.

13. Instituto Brasileiro de Geografia e Estatística (IBGE). Pesquisa Nacional de Saúde 2013: percepção do estado de saúde, estilos de vida e doenças crônicas: Brasil, Grandes Regiões e Unidades da Federação [Internet]. Rio de Janeiro: IBGE; 2014 [acessado 2016 Jun 9]. Disponível em: http://biblioteca.ibge.gov.br/visualizacao/livros/ liv91110.pdf

14. Prado MAMB, Francisco PMSB, Barros MBA. Diabetes em idosos: uso de medicamentos e risco de interação medicamentosa. Cien Saude Colet 2016; 21(11):34473458.
15. Stopa SR, César CLG, Segri NJ, Goldbaum M, Guimarães VMV, Alves MCGP, Barros MBA. Diabetes autorreferido em idosos: comparação das prevalências e medidas de controle. Rev Saude Publica 2014; 48(4):554562.

16. Francisco PMSB, Belon AP, Barros MBA, Carandina L, Alves MCGP, Goldbaum M, Cesar CLG. Diabetes autorreferido em idosos: prevalência, fatores associados e práticas de controle. Cad Saude Publica 2010; 26(1):175-184.

17. Öztürk A, Sim ek TT, Yümin ET, Sertel M, Yümin M. The relationship between physical, functional capacity and quality of life (QoL) among elderly people with a chronic disease. Arch Gerontol Geriatr 2011; 53(3):278283.

18. Malta DC, Iser BPM, Chueiri PS, Stopa SR, Szwarcwald CL, Schmidt MI, Duncan BB. Cuidados em saúde entre portadores de diabetes mellitus autorreferido no Brasil, Pesquisa Nacional de Saúde, 2013. Rev. bras. epidemiol. 2015; 18(Supl. 2):17-32.

19. Campolina AS, Adami F, Santos JLF, Lebrão ML. A transição de saúde e as mudanças na expectativa de vida saudável da população idosa: possíveis impactos da prevenção de doenças crônicas. Cad Saude Publica 2013; 29(6):1217-1229.

20. Bonow RO, Mitch WE, Nesto RW, O'Gara PT, Becker RC, Clark LT, Hunt S, Jialal I, Lipshultz SE, Loh E. Prevention conference VI. Diabetes and Cardiovascular disease. Writing Group V: Management of Cardiovascular-Renal Complications. Circulation 2002; 105(18):e159-164.

21. Kannel WB. Risk stratification in hypertension: new insights from the Framingham Study. Am J Hypertens 2000; 13(1 Pt 2):3S-10S

22. Hu G, Jousilahti P, Tuomilehto J. Joint effects of history of hypertension at baseline and type 2 diabetes at baseline and during follow-up on the risk of coronary heart disease. Eur Heart J 2007; (28):3059-3066.

23. Instituto Brasileiro de Geografia e Estatística (IBGE). Síntese de indicadores sociais 2015: uma análise das condições de vida da população brasileira. [acessado 2016 Jul 3]. Disponível em: http://biblioteca.ibge.gov.br/visualizacao/livros/liv95011.pdf

24. World Health Organization (WHO). A conceptual framework for action on the social determinants of health. Geneva: WHO; 2010.

25. Brasil. Ministério da Saúde (MS). Vigitel Brasil 2012: vigilância de fatores de risco e proteção para doenças crônicas por inquérito telefônico. Brasília: MS; 2013.

26. Cervi A, Franceschini SCC, Priore SE. Análise do uso do índice de massa corporal para idosos. Rev Nutr 2005; 18(6):765-775.

27. Instituto Brasileiro de Geografia e Estatística (IBGE). Pesquisa Nacional por Amostra de Domicílios: um panorama da saúde no Brasil - acesso e utilização dos serviços, condições de saúde e fatores de risco e proteção à saúde 2008. Rio de Janeiro: IBGE; 2010.

28. Snijders TAB, Bosker RJ. Multilevel analysis. An in troduction to basic and advanced multilevel modelling. London: Sage Publications; 1999. 
29. Lebrão ML, Laurenti R. Saúde, bem-estar e envelhecimento: o estudo SABE no Município de São Paulo. Rev Bras Epidemiol 2005; 8(2):127-141.

30. Lima-Costa MF, Matos DL, Camargos VP, Macinko J. Tendências em dez anos das condições de saúde de idosos brasileiros: evidências da Pesquisa Nacional por Amostra de Domicílios (1998, 2003, 2008). Cien Saude Colet 2011; 16(9):3689-3696.

31. Fried L, Tangen C, Walston J, Newman A, Hirsch C, Gottdiener J, Seeman T, Tracy R, Kop WJ, Burke G, McBurnie MA; Cardiovascular Health Study Collaborative Research Group. Frailty in older adults: evidence for a phenotype. J Gerontol A Biol Sci Med Sci 2001; 56(3):M146-156.

32. Freitas LRS, Garcia LP. Evolução da prevalência do diabetes e deste associado à hipertensão arterial no Brasil: análise da Pesquisa Nacional por Amostra de Domicílios, 1998, 2003 e 2008. Epidemiol Serv Saude 2012; 21(1):7-19.

33. Silva DB, Souza TA, Santos CM, Jucá MM, Moreira TMM, Frota MA, Vasconcelos SMM. Associação entre hipertensão arterial e diabetes em centro de saúde da família. Revista Brasileira em Promoção da saúde 2011; 24(1):16-23.

34. Loyola Filho AI, Firmo JOA Uchoa E, Lima-Costa MF. Fatores associados à autoavaliação negativa da saúde entre idosos hipertensos e/ou diabéticos: resultados do projeto Bambuí. Rev. bras. Epidemiol. 2013; 16(3):559571.

35. Barata RB. Desigualdades sociais e saúde. Campos GWS, Bonfim JRA, Minayo MCS, Akerman M, Júnior MD, Carvalho YM, organizadores. Tratado de Saúde Coletiva. São Paulo: Hucitec; 2014. p. 457-486.

36. Ullits LR, Ejlskov L, Mortensen RN, Hansen SM, Kræmer SR, Vardinghus-Nielsen H, Fonager K, Bøggild $\mathrm{H}$, Torp-Pedersen C, Overgaard C. Socioeconomic inequality and mortality - a regional Danish cohort study. BMC Public Health 2015; 15:490.

37. Ng JH, Bierman AS, Elliott MN, Wilson RL, Xia C, Scholle SH. Beyond black and white: race/ethnicity and health status among older adults. Am J Manag Care 2014; 20(3):239-248.

38. Brasil. Ministério da Saúde (MS). Plano de ações estratégicas para o enfrentamento das doenças crônicas não transmissiveis (DCNT) no Brasil 2011-2022. Brasília: MS; 2011 [acessado em 2013 Jul 05]. Disponível em: http://bvsms.saude.gov.br/bvs/publicacoes/plano_ acoes_enfrent_dcnt_2011.pdf

39. Laguardia J. Raça, genética \& hipertensão: nova genética ou velha eugenia? História, Ciências, Saúde Manguinhos 2005; 12(2):371-393.

40. Marinho V, Blay SL, Andreoli SB, Gastal F. A prevalence study of current tobacco smoking in later life community and its association with sociodemographic factors, physical health and mental health status. Soc Psychiatry Psychiatr Epidemiol 2008; 43(6):490-497.
41. Zaitune MPA, Barros MBA, Lima MG, César CLG, Carandina L, Goldbaum M, Alves MCGP. Fatores associados ao tabagismo em idosos: Inquérito de Saúde no Estado de São Paulo (ISA-SP). Cad Saude Publica 2012; 28(3):583-595.

42. Lima Costa MFF, Peixoto SV, César CC, Malta DC, Moura EC. Comportamentos em saúde entre idosos hipertensos, Brasil, 2006. Rev Saude Publica 2009; 43(Supl. 2):18-26.

43. World Health Organization (WHO). Global estimate of the burden of disease from second-hand smoke. Geneva: WHO; 2010.

44. Reichert J, Araújo AJ, Gonçalves CMC, Godoy I, Chatkin JM, Sales MPU, Santos SRRA. Diretrizes para cessação do tabagismo - 2008. J Bras Pneumol 2008; 34(10):845-880.

45. Kickbusch I. Promoción de la salud: Una pespectiva mundial. In: Organización Panamericana de la Salud (OPAS). Promoción de la Salud: una antologia. Washington: OPAS; 1996. Publicação Científica 557. p. 15-24.

46. Fundação Oswaldo Cruz (Fiocruz). A saúde no Brasil em 2030: diretrizes para a prospecção estratégica do Sistema de Saúde Brasileiro. Rio de Janeiro: Fiocruz; 2012.

47. Geib LTC. Determinantes sociais da saúde do idoso. Cien Saude Colet 2012; 17(1):123-133.

48. Goldstein H. Multilevel statistical models. $3^{\text {th }}$ ed. London: Edward Arnold; 2003.

49. Lima-Costa MF, Peixoto SV, Firmo JOA. Validade da hipertensão arterial auto-referida e seus determinantes (projeto Bambuí). Rev Saude Publica 2004; 38(5):637642.

50. Selem SSC, Castro MA, César CLG, Marchioni DML, Fisberg RM. Validade da hipertensão autorreferida associa-se inversamente com escolaridade em brasileiros. Arq Bras Cardiol 2013; 100(1):52-59.

51. Chrestani MA, Santos IS, Matijasevich AM. Hipertensão arterial sistêmica auto-referida: validação diagnóstica em estudo de base populacional. Cad Saude Publica 2009; 25(11):2395-406.

52. Bernal R, Silva NN. Cobertura de linhas telefônicas residenciais e vícios potenciais em estudos epidemiológicos. Rev Saude Publica 2009; 43(3):421-426.

53. Francisco PMSB, Barros MBA, Segri NJ, Alves MCGP, Cesar CLG, Malta DC. Comparação de estimativas para o auto-relato de condições crônicas entre inquérito domiciliar e telefônico - Campinas (SP), Brasil. Rev Bras Epidemiol 2011; 14(1):5-15.

Artigo apresentado em 25/08/2016

Aprovado em 09/11/2016

Versão final apresentada em 11/11/2016 